\title{
A subanalysis of Japanese patients in a randomized, double- blind, placebo-controlled, phase 3 trial of nivolumab for patients with advanced gastric or gastro-esophageal junction cancer refractory to, or intolerant of, at least two previous chemotherapy regimens (ONO-4538-12, ATTRACTION-2)
}

\author{
Ken Kato ${ }^{1} \cdot$ Taroh Satoh $^{2} \cdot$ Kei Muro $^{3} \cdot$ Takaki Yoshikawa $^{4,32} \cdot$ Takao Tamura $^{5,33} \cdot$ Yasuo Hamamoto $^{6} \cdot$ Keisho Chin $^{7}$. \\ Keiko Minashi ${ }^{8} \cdot$ Masahiro Tsuda $^{9} \cdot K$ Kensei Yamaguchi ${ }^{10,34} \cdot$ Nozomu Machida $^{11}$. Taito Esaki ${ }^{12}$ - Masahiro Goto ${ }^{13}$. \\ Yoshito Komatsu ${ }^{14} \cdot$ Takako Eguchi Nakajima $^{15} \cdot$ Naotoshi Sugimoto $^{16} \cdot$ Kazuhiro Yoshida $^{17} \cdot$ Eiji Oki $^{18}$.

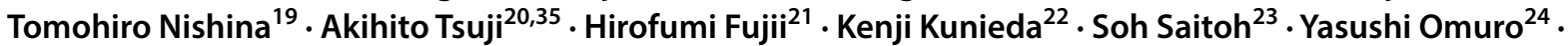 \\ Mizutomo Azuma ${ }^{25}$. Yasuo Iwamoto ${ }^{26} \cdot$ Keisei Taku $^{27}$ - Sachio Fushida ${ }^{28} \cdot$ Li-Tzong Chen $^{29,30} \cdot$ Yoon-Koo Kang ${ }^{31}$. \\ Narikazu Boku' ${ }^{1}$
}

Received: 22 August 2018 / Accepted: 12 November 2018 / Published online: 1 December 2018

(c) The Author(s) 2018

\begin{abstract}
Background Nivolumab, an anti-programmed death-1 agent, showed survival benefits in Asian patients, including Japanese, with gastric/gastro-esophageal junction (G/GEJ) cancer. We report the analysis of the Japanese subpopulation from ATTRACTION-2 that evaluated nivolumab versus placebo in unresectable advanced or recurrent G/GEJ cancer after $\geq 2$ chemotherapy regimens.

Methods Data from the Japanese subpopulation in the randomized, double-blind, placebo-controlled, phase 3 trial were analyzed (data cutoff, February 25, 2017). Primary endpoint was overall survival (OS); secondary endpoints included progression-free survival (PFS) and objective response rate (ORR).

Results Among the overall study population of 493 patients, 226 (nivolumab 152; placebo 74) were enrolled from 28 sites in Japan. In the Japanese subset, median OS was longer with nivolumab versus placebo (5.4 months, 95\% CI 4.6-7.4 versus 3.6 months, $95 \%$ CI 2.8-5.0). The risk of death was lower in the nivolumab versus placebo group (hazard ratio $0.58,95 \%$ CI $0.42-0.78 ; p=0.0002)$. Incidences of serious adverse events were $23 \%(35 / 152)$ and $25 \%(18 / 72)$ in the nivolumab and placebo groups, respectively. In the Japanese ITT population, $22 \%$ of nivolumab-treated and $28 \%$ of placebo-treated patients received prior ramucirumab treatment. Overall, clinical activity of nivolumab was observed regardless of prior ramucirumab use. In the nivolumab group, ORR and PFS were numerically higher in patients with prior ramucirumab use than in those without.

Conclusions In the Japanese subpopulation, patients receiving nivolumab had longer OS, similar to the overall population, with a manageable safety profile. The interaction between nivolumab and ramucirumab will be clarified in ongoing clinical trials.
\end{abstract}

Keywords Gastric cancer · Gastro-esophageal junction cancer · Japan · Nivolumab

Electronic supplementary material The online version of this article (https://doi.org/10.1007/s10120-018-0899-6) contains supplementary material, which is available to authorized users.

Narikazu Boku

nboku@ncc.go.jp

Extended author information available on the last page of the article

\section{Introduction}

In Japan, gastric cancer was the third leading cause of cancer-related death in 2016 and the most common malignancy in 2013 [1]. In general, treatment options include cytotoxic chemotherapy with addition of biologics for advanced gastric cancer. Since cross-over use of paclitaxel and irinotecan 
in second- and third-line chemotherapy was considered to contribute to a favorable overall survival (OS) in the WJOG 4007 study [2] compared with other studies outside Japan, irinotecan monotherapy is recommended as third-line therapy in the 2018 Gastric Cancer Treatment Guidelines by the Japanese Gastric Cancer Association (evidence level B) [3]. Regardless of these treatment options from first- to thirdline treatment, the prognosis for advanced gastric cancer patients is poor, with a median OS of 13-14 months [4, 5]. Therefore, development of new therapies for patients with advanced gastric or gastro-esophageal junction (G/GEJ) cancer is warranted.

Inhibition of immune checkpoints is a proven therapeutic approach for many cancers. It includes the receptor-ligand system targeting programmed death-1 (PD-1), which is a cell surface receptor that blocks antitumor T-cell activity [6] after binding with programmed death-ligand 1 (PD-L1), which is expressed in $25-65 \%$ of gastric cancers and associated with tumor size, lymph node metastasis, and a shorter median survival [7-9]. Immuno-oncology agents, which block binding of PD-1 and PD-L1, are also being evaluated for G/GEJ cancer. The phase 1/2 CheckMate 032 trial reported clinical activity of nivolumab, alone or in combination with ipilimumab, an anti-cytotoxic T-lymphocyte-associated protein-4 antibody, in the gastric cohort with chemotherapyrefractory advanced $\mathrm{G} / \mathrm{GEJ} /$ esophageal cancer $[10,11]$. The phase 3 ATTRACTION-2 (ONO-4538-12) study conducted in Japan, Taiwan, and South Korea was also designed to investigate the efficacy and safety of nivolumab in heavily pretreated patients unselected for PD-L1 tumor expression. The results showed survival benefit with nivolumab versus placebo (median OS, 5.3 months vs 4.1 months; 12-month survival rates, $26.2 \%$ vs $10.9 \%$, respectively), indicating that nivolumab can be a new treatment option for heavily pretreated patients with advanced G/GEJ cancer [12]. Therefore, nivolumab has been added as recommended third- or later-line therapy in the 2018 guideline (evidence level A) based on the prolonged OS in patients with advanced gastric cancer with failure of $\geq 2$ lines of chemotherapy (ATTRAC TION-2 study) [3, 12]. Further, the National Comprehensive Cancer Network guideline also recommends pembrolizumab as a third-line or subsequent therapy for recurrent, unresectable locally advanced, or metastatic gastric adenocarcinoma with PD-L1 expression [13].

However, the treatment strategy for advanced gastric cancer in Japan is somewhat different from that in Taiwan and South Korea. In Japan, the anti-vascular endothelial growth factor (VEGF) receptor 2 antibody ramucirumab, which is covered by National Health Insurance since 2015, has been widely used in combination with paclitaxel in second-line therapy. Thereafter, third-line chemotherapy is common if the patient's condition is good. Several papers have reported that inhibition of VEGF signals changes the tumor microenvironment [14-18], which might have some influence on nivolumab efficacy. Therefore, we additionally analyzed data from the Japanese subpopulation to explore the impact of prior ramucirumab use on the efficacy of nivolumab.

\section{Methods}

\section{Study design and patients}

Data from Japanese patients enrolled in the randomized, double-blind, placebo-controlled, phase 3 ATTRACTION-2 trial (49 clinical sites in Japan, South Korea, and Taiwan) were analyzed. As a follow-up/update of ATTRACTION-2, the data cutoff date was February 25, 2017 for the Japanese subpopulation. The study design and results from the overall population have been previously reported [12].

\section{Procedures}

Patients received nivolumab $3 \mathrm{mg} / \mathrm{kg}$ or placebo infusion every 2 weeks for each 6 -week cycle (three infusions per 6 -week cycle). Tumor assessments were performed after every 6-week treatment cycle for ten cycles (approximately 14 months). Thereafter, tumor assessments were performed after every two treatment cycles (12 weeks) until discontinuation of study treatment. A final assessment was also performed at the end-of-treatment examination. All tumor assessments were performed using computed tomography or magnetic resonance imaging according to the Response Evaluation Criteria in Solid Tumors guidelines version 1.1 [19]. The details have been described previously [12].

Adverse events (AEs) were assessed according to the National Cancer Institute Common Terminology Criteria for AEs version 4.0 [20] during the treatment period and for 28 days after end of treatment.

Tumor tissue collection was not compulsory; available tumor samples, which were collected at baseline, were evaluated retrospectively for PD-L1 tumor expression. Tumors with immunohistochemical staining in $\geq 1 \%$ of tumor cells, as assessed at a central laboratory (28-8 pharmDx assay; Dako, Carpinteria, CA, USA), were defined as positive.

\section{Outcomes}

The primary endpoint was OS. Secondary efficacy endpoints included progression-free survival (PFS), objective response rate (ORR; the proportion of patients with confirmed complete response [CR] and partial response [PR]), disease control rate (DCR; the proportion of patients with confirmed CR, PR, and stable disease [SD]), duration of response (DOR), time to response, best overall response 
(CR, PR, SD, and progressive disease [PD]), and maximum percentage change from baseline in the sum of diameters of target lesions (\% tumor shrinkage). PD-L1 expression status in patients with available tumor tissue sample was evaluated as an exploratory analysis. Safety endpoints included AEs and treatment-related AEs.

\section{Statistical analysis}

Survival analyses were performed in the intention-to-treat (ITT) population, defined as all patients who were randomly assigned to the study treatment. Response rate was calculated in patients with measurable target lesions at baseline (response assessment population). Safety analyses were completed using data from all patients who received at least one dose of study treatment (safety population). The estimations of the OS and PFS rates were derived from the Kaplan-Meier estimates and the corresponding confidence intervals (CIs) were derived based on the Greenwood formula for variance and on log-log transformation. OS and PFS were compared using the stratified log-rank test with a one-sided significance level of 0.025 . For best overall response, the exact $95 \%$ CI was calculated using the Clopper-Pearson method. Calculation of $p$ value was conducted using the Cochran-Mantel-Haenszel test; SAS software (versions 9.3 and 9.4) was used for all statistical analyses. Other information has been reported previously [12].

\section{Results}

\section{Patient disposition and baseline characteristics}

Overall, 226 (nivolumab 152; placebo 74) of the 493 patients in ATTRACTION-2 were enrolled from 28 study sites in Japan. The safety population comprised 224 patients (nivolumab 152; placebo 72), and the response assessment population comprised 189 patients (nivolumab 129; placebo 60) (Supplementary Fig. 1). Baseline characteristics of the Japanese patients were well balanced between the treatment arms (Table 1). A total of 55 patients (nivolumab 34; placebo 21) were treated with ramucirumab prior to study entry.

\section{Exposure and subsequent pharmacotherapy in the Japanese subpopulation}

The median (range [min-max]) duration of treatment was $2.2(0.0-24.4)$ months with nivolumab and $1.0(0.0-26.3)$ months with placebo. Overall, the relative dose intensity of nivolumab was $\geq 90 \%$ to $<110 \%$ in $82.9 \%$ of patients. Details of study drug exposure and administration are presented in Supplementary Table 1. At data cutoff, study treatment was permanently discontinued in 143 patients in the
Table 1 Baseline patient characteristics of subgroup of Japanese patients

\begin{tabular}{|c|c|c|}
\hline & $\begin{array}{l}\text { Nivolumab } \\
3 \mathrm{mg} / \mathrm{kg}(N=152)\end{array}$ & $\begin{array}{l}\text { Placebo } \\
(N=74)\end{array}$ \\
\hline Male & $111(73)$ & $57(77)$ \\
\hline Female & $41(27)$ & $17(23)$ \\
\hline Age (years) median (min, max) & $65(20,83)$ & $66(28,79)$ \\
\hline Patients aged $<65$ years & $68(45)$ & $28(38)$ \\
\hline \multicolumn{3}{|l|}{ Country } \\
\hline Japan & 152 & 74 \\
\hline Korea & - & - \\
\hline Taiwan & - & - \\
\hline \multicolumn{3}{|c|}{ Eastern Cooperative Oncology Group performance status } \\
\hline 0 & $64(42)$ & $31(42)$ \\
\hline 1 & $88(58)$ & $43(58)$ \\
\hline \multicolumn{3}{|l|}{ Organs with metastases } \\
\hline$<2$ & $43(28)$ & $22(30)$ \\
\hline$\geq 2$ & $109(72)$ & $52(70)$ \\
\hline \multicolumn{3}{|l|}{ Site of metastases } \\
\hline Lymph node & $129(85)$ & $60(81)$ \\
\hline Peritoneum & $28(18)$ & $15(20)$ \\
\hline Liver & $35(23)$ & $17(23)$ \\
\hline Lung & $11(7)$ & $4(5)$ \\
\hline Pleura & $1(1)$ & $1(1)$ \\
\hline Adrenal glands & 0 & $2(3)$ \\
\hline Bone & $3(2)$ & $3(4)$ \\
\hline Other & $8(5)$ & $7(10)$ \\
\hline \multicolumn{3}{|l|}{ Previous treatment regimens ${ }^{\mathrm{a}}$} \\
\hline 2 & $11(7)$ & $3(4)$ \\
\hline 3 & $57(38)$ & $26(35)$ \\
\hline$\geq 4$ & $84(55)$ & $45(61)$ \\
\hline \multicolumn{3}{|l|}{ Previous therapies } \\
\hline Any & $152(100)$ & $74(100)$ \\
\hline Pyrimidine analogs & $152(100)$ & $74(100)$ \\
\hline Platinum & $138(91)$ & $71(96)$ \\
\hline Taxanes & $150(99)$ & $72(97)$ \\
\hline Irinotecan & $137(90)$ & $70(95)$ \\
\hline Ramucirumab & $34(22)$ & $21(28)$ \\
\hline \multicolumn{3}{|l|}{ Previous gastrectomy } \\
\hline No & $56(37)$ & $31(42)$ \\
\hline Yes & $96(63)$ & $43(58)$ \\
\hline
\end{tabular}

Data shown are $n(\%)$ unless otherwise stated

${ }^{a}$ Includes treatments received in the adjuvant setting

nivolumab group and in 71 patients in the placebo group. Reasons for treatment discontinuation in the nivolumab versus placebo group were disease progression (114 [75\%] vs 53 [73.6\%]), worsening of clinical symptoms judged as PD (32 [21.1\%] vs 17 [23.6\%]), onset of grade $\geq 2$ interstitial lung disease (4 [2.6\%] vs 0 [0\%]), physician's discretion (6 [3.9\%] vs $2[2.8 \%])$, treatment withheld for $>6$ weeks due 
to AEs (2 [1.3\%] vs 1 [1.4\%]), and other reasons (4 [2.6\%] vs $5[6.9 \%])$, respectively.

Following study treatment discontinuation, 53.3\% (81/152) and 44.6\% (33/74) of patients in the nivolumab and placebo groups, respectively, received subsequent anticancer treatment (pharmacotherapy, 30.9\% [47/152] vs $24.3 \%$ [18/74]; surgery, $19.7 \%$ [30/152] vs $10.8 \%$ [8/74]; and radiotherapy, 9.2\% [14/152] vs $13.5 \%$ [10/74], respectively; Supplementary Table 2).

\section{Efficacy in the Japanese subpopulation}

\section{Overall survival}

In the Japanese ITT population, as of February 25, 2017, $186(82.3 \%)$ deaths had occurred (nivolumab 120 [78.9\%]; placebo 66 [89.2\%]). Median follow-up in the surviving patients was 16.6 months (interquartile range [IQR] $13.0-20.6 ; n=32$ ) in the nivolumab group and 17.7 months (IQR 13.2-25.8; $n=8$ ) in the placebo group. By Kaplan-Meier analysis, the median OS was longer in the nivolumab versus placebo group (5.4 months, 95\% CI 4.6-7.4 versus 3.6 months, 95\% CI 2.8-5.0; Fig. 1a; Table 2). The risk of death was significantly lower in the nivolumab versus placebo group (hazard ratio [HR] 0.58, 95\% CI 0.42-0.78; $p=0.0002)$. The OS rates were higher in the nivolumab versus placebo group at 6,12 , and 18 months (Table 2). Subgroup analyses of OS according to selected disease characteristics consistently favored nivolumab over placebo (Supplementary Fig. 2).

\section{Progression-free survival}

By Kaplan-Meier analysis, PFS was significantly longer in the nivolumab versus placebo group at all follow-up time points (Fig. 1b; Table 2). The median PFS was 1.7 months (95\% CI 1.6-2.8) in the nivolumab group versus 1.5 months (95\% CI 1.5-1.6) in the placebo group. The 6-month PFS rate was higher in the nivolumab versus placebo group (19.0\%, 95\% CI $13.0-25.9$ versus $6.2 \%, 95 \%$ CI $2.0-13.7)$. The risk of disease progression was lower in the nivolumab versus placebo group (HR 0.53 , 95\% CI $0.39-0.72$; $p<0.0001)$.

\section{Response}

Patients with a confirmed response to nivolumab showed a median time to response of 1.7 months (min-max, 1.4-7.0). The median DOR was 14.5 months $(95 \%$ CI 8.3-not available [NA]), and ORR was $14.0 \%(18 / 129)$ in patients treated with nivolumab. DCR was $45.0 \%$ (58/129 patients) in the nivolumab group versus $23.3 \%$ (14/60 patients) in the placebo group (odds ratio 2.87, 95\% CI 1.40-5.88; Table 3 and Supplementary Fig. 3).

\section{PD-L1 expression status}

Baseline tumor samples were available for $61 \%(93 / 152)$ of patients in the nivolumab group and for 55\% (41/74) of patients in the placebo group. PD-L1 expression was quantifiable in 91 and 41 patient samples in the nivolumab and placebo groups, respectively. Among them $13.2 \%$ (12/91) of patients in the nivolumab group and $19.5 \%(8 / 41)$ of patients in the placebo group had PD-L1-positive tumors. Although the patient numbers were low and the results did not reach significance, benefits of nivolumab (median OS, 6.14 months) were observed even in patients without PD-L1-positive tumors (HR 0.76, 95\% CI 0.49-1.18) as compared to the median OS reported in this study.

\section{Safety in the Japanese subpopulation}

Overall, AEs were reported more frequently in the nivolumab versus placebo group (Supplementary Table 3). All-cause AEs of any grade occurred in $84.9 \%$ (129/152) of patients in the nivolumab group versus $73.6 \%$ (53/72) in the placebo group; incidences of serious AEs were 23\% (35/152) versus 25\% (18/72), respectively.

Treatment-related AEs of any grade were reported in $56.6 \%$ (86/152) of patients in the nivolumab group versus $30.6 \%$ (22/72) in the placebo group; grade 3/4 treatment-related AEs were $15.8 \%$ (24/152) versus $9.7 \%$ (7/72), respectively. Incidences of serious treatment-related AEs were 13.2\% (20/152) versus $9.7 \%$ (7/72), respectively.

The most commonly ( $>5 \%$ incidence) reported all-grade treatment-related AEs were pruritus, diarrhea, rash, fatigue, nausea, malaise, and decreased appetite. The most commonly reported grade 3/4 AEs were decreased appetite and diarrhea in the nivolumab group and decreased appetite and fatigue in the placebo group (Table 4). The incidence of most frequent treatment-related serious AEs was low $(\leq 2 \%)$ in the nivolumab group and included interstitial lung disease, type 1 diabetes mellitus, and colitis (Supplementary Table 4). Two deaths (1.3\%; cardiac arrest, unknown cause) in the nivolumab group versus 1 death (1.4\%; gastrointestinal perforation) in the placebo group were considered treatment-related AEs in the Japanese subpopulation. Treatment was discontinued due to treatment-related AEs in $4.6 \%$ (7/152) versus 5.6\% (4/72) of patients in the nivolumab versus placebo group, respectively.

\section{Efficacy analysis of patients with prior ramucirumab treatment}

A total of 34 and 21 patients received prior ramucirumab treatment in the nivolumab and placebo groups, respectively. 
Fig. 1 Kaplan-Meier plots of a overall survival and b progression-free survival (Japanese ITT population). $C I$ Confidence interval, $H R$ hazard ratio, ITT intention-to-treat

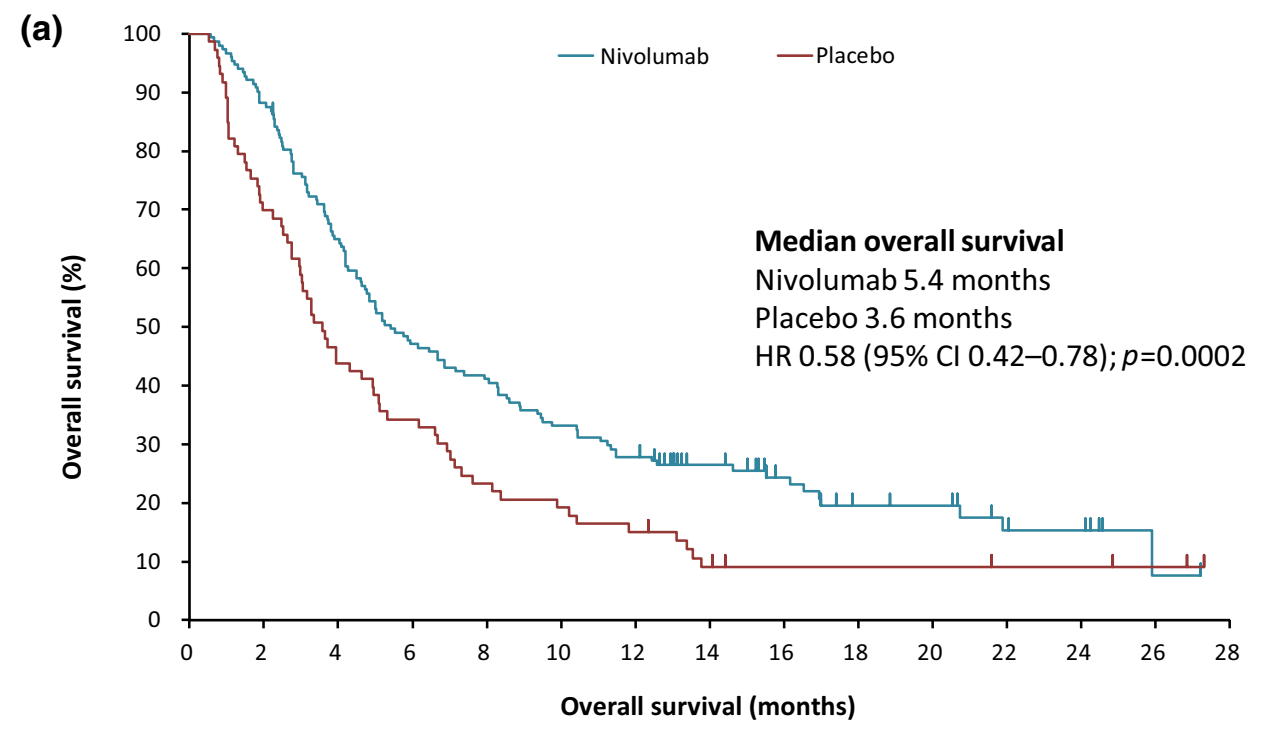

\begin{tabular}{|l|c|c|c|c|c|c|c|c|c|c|c|c|c|c|c|}
\hline At risk & 0 & 2 & 4 & 6 & 8 & 10 & 12 & 14 & 16 & 18 & 20 & 22 & 24 & 26 & 28 \\
\hline Nivolumab & 152 & 134 & 98 & 71 & 62 & 50 & 42 & 29 & 20 & 13 & 12 & 7 & 6 & 1 & 0 \\
\hline Placebo & 74 & 51 & 32 & 25 & 17 & 14 & 11 & 6 & 4 & 4 & 4 & 3 & 3 & 2 & 0 \\
\hline
\end{tabular}

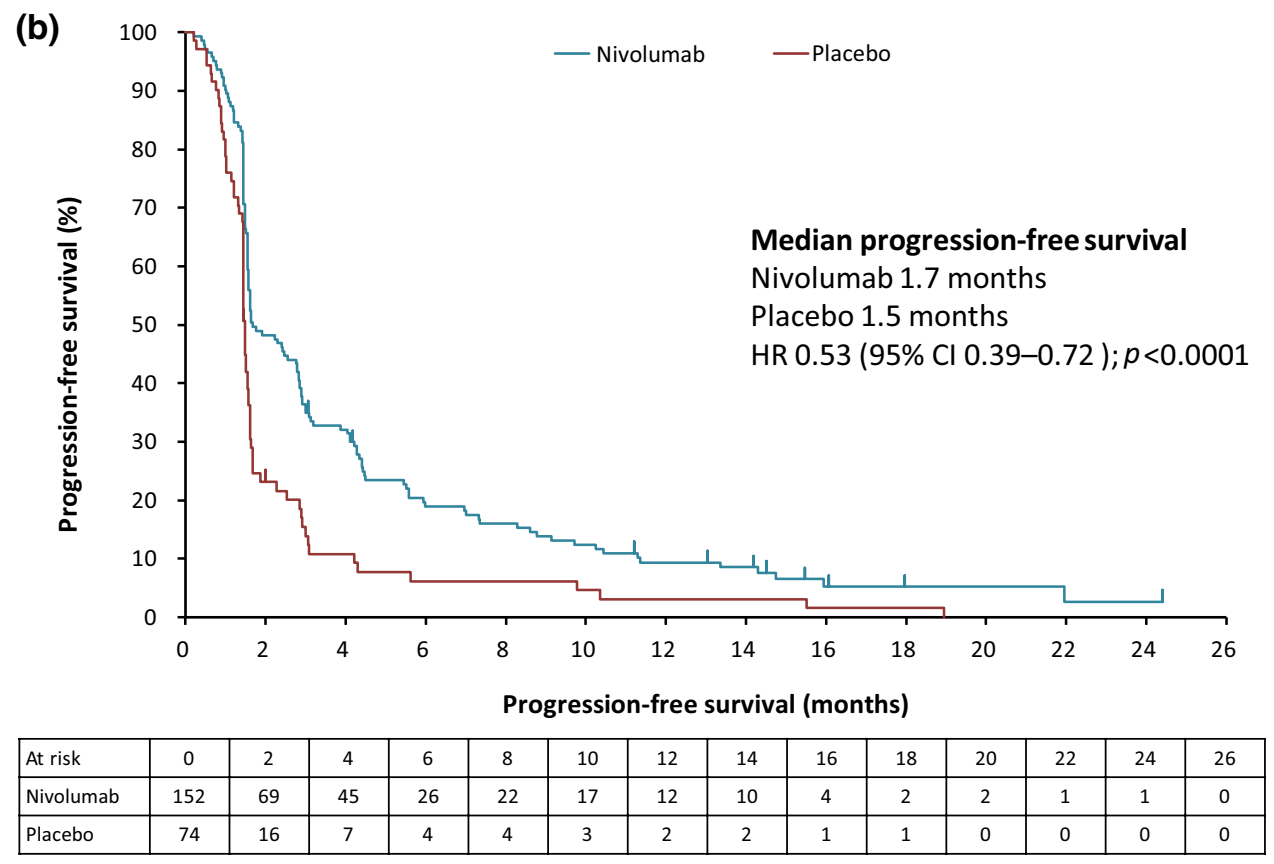

The baseline characteristics were similar in patients with and without prior ramucirumab use, except for the variability observed in the number of prior regimens (Supplementary Table 5). The median OS was longer in the nivolumab versus placebo group in patients with (5.3 versus 2.8 months [HR $0.57,95 \%$ CI $0.31-1.05]$ ) and without (5.5 versus 3.9 months [HR 0.65, 95\% CI 0.46-0.92]) prior ramucirumab treatment (Fig. 2a, b). The median PFS was longer in the nivolumab versus placebo group in patients with (3.0 versus 1.4 months [HR 0.39, 95\% CI 0.21-0.72]) and without (1.6 versus
1.5 months [HR $0.64,95 \%$ CI $0.45-0.90]$ ) prior ramucirumab treatment (Fig. 2c, d). Among patients evaluable for tumor response, the ORR in the nivolumab group was higher in patients with $(22.2 \%$ [6/27]) versus without $(11.8 \%$ [12/102]) prior ramucirumab treatment. DCR was higher in the nivolumab versus placebo group in patients with $(55.6 \%$ [15/27] vs $21.1 \%$ [4/19]) and without (42.2\% [43/102] vs $24.4 \%$ [10/41]) previous ramucirumab treatment (Table 5). Furthermore, better clinical activity was observed in 25 of the 34 patients who received nivolumab directly after 
Table 2 Median overall survival and progression-free survival rates at 3, 6, 9, 12, 18, and 24 months (Japanese ITT population)

\begin{tabular}{llc}
\hline & $\begin{array}{l}\text { Nivolumab 3 mg/kg } \\
(N=152)\end{array}$ & Placebo $(N=74)$ \\
\hline OS rate, \% (95\% CI) & & \\
At 3 months & $76.2(68.6-82.2)$ & $58.9(46.8-69.2)$ \\
At 6 months & $47.1(38.9-54.8)$ & $34.2(23.7-45.1)$ \\
At 9 months & $35.8(28.2-43.4)$ & $20.5(12.2-30.4)$ \\
At 12 months & $27.8(21.0-35.1)$ & $15.1(8.0-24.2)$ \\
At 18 months & $19.5(13.0-27.0)$ & $9.0(3.8-17.1)$ \\
PFS rate, \% (95\% CI) & & \\
At 3 months & $35.0(27.3-42.8)$ & $13.9(6.9-23.2)$ \\
At 6 months & $19.0(13.0-25.9)$ & $6.2(2.0-13.7)$ \\
At 9 months & $13.9(8.8-20.2)$ & $6.2(2.0-13.7)$ \\
At 12 months & $9.4(5.2-15.0)$ & $3.1(0.6-9.5)$ \\
At 18 months & $5.2(2.1-10.5)$ & $1.5(0.1-7.3)$ \\
\hline
\end{tabular}

The estimation of the OS rate was derived from the Kaplan-Meier estimate and corresponding CI was derived based on Greenwood formula for variance and on $\log -\log$ transformation

The estimation of the PFS rate was derived from the Kaplan-Meier estimate and corresponding CI was derived based on Greenwood formula for variance and on $\log -\log$ transformation

1 month $=30.4375$ days

CI Confidence interval, ITT, intention-to-treat, $O S$ overall survival, $P F S$ progression-free survival

ramucirumab treatment. In the ITT $(n=25)$ and response assessment $(n=20)$ populations, for patients who received nivolumab directly after ramucirumab, the median OS, median PFS, ORR, and DCR were 6.7 months, 4.2 months, $25.0 \%(5 / 20)$, and $70 \%(14 / 20)$, respectively.

\section{Discussion}

We report the analysis of data from the Japanese subpopulation in the randomized, double-blind, placebocontrolled, phase 3 ATTRACTION-2 trial. The results of this subanalysis showed consistency in the efficacy of nivolumab between the Japanese subpopulation and the overall study population reported previously [12]. Of note, more Japanese patients had received $\geq 4$ previous anticancer treatment regimens and their Eastern Cooperative Oncology Group (ECOG) performance status was better than that of the overall study population. In terms of efficacy, the OS, PFS, DOR, SD, and DCR were similar between the Japanese subpopulation and the overall study population, in both the nivolumab and placebo groups. The risks of death and disease progression were lower in the nivolumab group than in the placebo group and this was consistent throughout the follow-up period in both populations. Subgroup analyses of OS according to selected
Table 3 Best overall response in Japanese subpopulation (response assessment population)

\begin{tabular}{lll}
\hline & Nivolumab $(N=129)$ & Placebo $(N=60)$ \\
\hline Best overall response, $n(\%)$ & & \\
CR & 0 & 0 \\
PR & $18(14.0)$ & 0 \\
SD & $40(31.0)$ & $14(23.3)$ \\
PD & $61(47.3)$ & $40(66.7)$ \\
NE & $10(7.8)$ & $6(10.0)$ \\
ORR & & \\
ORR $(\mathrm{CR}+\mathrm{PR})$ & $18(14.0)$ & 0 \\
$(95 \% \mathrm{CI})^{\mathrm{a}}$ & $(8.5,21.2)$ & $(0.0,6.0)$ \\
$p$ value & \\
DCR & $0.0023^{\mathrm{b}}$ & \\
DCR $(\mathrm{CR}+\mathrm{PR}+\mathrm{SD})$ & $58(45.0)$ & $14(23.3)$ \\
$(95 \% \mathrm{CI})^{\mathrm{a}}$ & $(36.2-54.0)$ & $(13.4-36.0)$ \\
$p$ value $^{\mathrm{b}}$ & $0.0037^{*}$ & \\
\hline
\end{tabular}

Best overall response was determined solely by imaging assessment according to the RECIST Guideline Version 1.1

$C I$ Confidence interval, $C R$ complete response, DCR disease control rate, ECOG Eastern Cooperative Oncology group, $N E$, not evaluable, $O R R$ objective response rate, $P D$ progressive disease, $P R$ partial response, $S D$ stable disease

$* p<0.05$

${ }^{\text {a }}$ Exact $95 \%$ CI was calculated using Clopper-Pearson method

${ }^{\mathrm{b}}$ The calculation of $p$ value was conducted using Cochran-MantelHaenszel test adjusted by the following three factors (interactive web response system): (1) location (Japan versus Korea versus Taiwan); (2) ECOG performance status score at baseline (0 versus 1); (3) number of organs with metastases $(<2$ vs $\geq 2)$

disease characteristics were also similar in both populations and consistently favored nivolumab over placebo (Supplementary Fig. 2).

A greater median OS has previously been reported with the biological agent ramucirumab as second-line treatment in Japanese/East Asian patients compared with Western patients with gastric cancer [21]. Furthermore, OS benefits with new agents over control treatments observed in nonJapanese/non-Asian populations were not confirmed in the Japanese/Asian subpopulation [21, 22]. It is considered that the high rate of post-discontinuation therapy (second-line or later) in the Japanese subpopulation does not allow differentiation of efficacy with new agents in these trials. Considering this difference in treatment strategy and clinical course, the significant improvement in OS and PFS with nivolumab, compared with the placebo group, in the Japanese subpopulation and East Asian patients from ATTRACTION-2 was a noteworthy outcome. Of note, although the Japanese subpopulation of ATTRACTION-2 received treatment in a lateline setting, the favorable outcome with nivolumab may be partly attributed to the better performance status of these patients, with $42 \%$ having an ECOG performance status of 0 . 
Table 4 Incidence of treatmentrelated adverse events occurring in $\geq 5 \%$ of Japanese patients and additional treatment-related adverse events of special interest (safety population)
Table 5 Best overall response in Japanese subpopulation based on prior treatment with ramucirumab (response assessment population)

\begin{tabular}{|c|c|c|c|c|}
\hline \multirow[t]{2}{*}{ Adverse event, $n(\%)$} & \multicolumn{2}{|c|}{ Nivolumab $(N=152)$} & \multicolumn{2}{|c|}{ Placebo $(N=72)$} \\
\hline & Any grade & Grade 3/4 & Any grade & Grade 3/4 \\
\hline All & $86(56.6)$ & $24(15.8)$ & $22(30.6)$ & $7(9.7)$ \\
\hline Pruritus & $17(11.2)$ & 0 & $1(1.4)$ & 0 \\
\hline Diarrhea & $14(9.2)$ & $2(1.3)$ & $2(2.8)$ & 0 \\
\hline Malaise & $12(7.9)$ & 0 & $6(8.3)$ & 0 \\
\hline Fatigue & $11(7.2)$ & $1(0.7)$ & $4(5.6)$ & $2(2.8)$ \\
\hline Decreased appetite & $10(6.6)$ & $3(2.0)$ & $4(5.6)$ & $1(1.4)$ \\
\hline Rash & $10(6.6)$ & 0 & $2(2.8)$ & 0 \\
\hline Nausea & $10(6.6)$ & 0 & $1(1.4)$ & 0 \\
\hline \multicolumn{5}{|c|}{ Additional treatment-related adverse events of special interest } \\
\hline Interstitial lung disease & $6(3.9)$ & $1(0.7)$ & 0 & 0 \\
\hline Rash maculo-papular & $5(3.3)$ & 0 & 0 & 0 \\
\hline Colitis & $2(1.3)$ & $1(0.7)$ & 0 & 0 \\
\hline Hypopituitarism & $1(0.7)$ & $1(0.7)$ & 0 & 0 \\
\hline Pneumonitis & $1(0.7)$ & $1(0.7)$ & 0 & 0 \\
\hline Hyperthyroidism & $1(0.7)$ & 0 & 0 & 0 \\
\hline Thyroid disorder & $1(0.7)$ & 0 & 0 & 0 \\
\hline Hepatic function abnormal & 0 & 0 & $2(2.8)$ & $1(1.4)$ \\
\hline Acute hepatic failure & 0 & 0 & $1(1.4)$ & $1(1.4)$ \\
\hline Acute hepatitis & 0 & 0 & 0 & 0 \\
\hline Autoimmune thyroiditis & 0 & 0 & 0 & 0 \\
\hline
\end{tabular}

\begin{tabular}{|c|c|c|c|c|}
\hline & \multicolumn{2}{|c|}{ With prior ramucirumab treatment } & \multicolumn{2}{|c|}{ Without prior ramucirumab treatment } \\
\hline & Nivolumab $(N=27)$ & Placebo $(N=19)$ & Nivolumab $(N=102)$ & Placebo $(N=41)^{\mathrm{a}}$ \\
\hline \multicolumn{5}{|c|}{ Best overall response, $n(\%)$} \\
\hline CR & 0 & 0 & 0 & 0 \\
\hline PR & $6(22.2)$ & 0 & $12(11.8)$ & 0 \\
\hline SD & $9(33.3)$ & $4(21.1)$ & $31(30.4)$ & $10(24.4)$ \\
\hline PD & $7(25.9)$ & $13(68.4)$ & $54(52.9)$ & $27(65.9)$ \\
\hline $\mathrm{NE}$ & $5(18.5)$ & $2(10.5)$ & $5(4.9)$ & $3(7.3)$ \\
\hline \multicolumn{5}{|l|}{ ORR } \\
\hline ORR (CR + PR) & $6(22.2)$ & 0 & $12(11.8)$ & 0 \\
\hline \multicolumn{5}{|l|}{ DCR } \\
\hline $\mathrm{DCR}(\mathrm{CR}+\mathrm{PR}+\mathrm{SD})$ & $15(55.6)$ & $4(21.1)$ & $43(42.2)$ & $10(24.4)$ \\
\hline
\end{tabular}

Best overall response was determined solely by imaging assessment according to the RECIST Guideline Version 1.1

$C R$ Complete response, $D C R$ disease control rate, $N E$ not evaluable, $O R R$ objective response rate, $P D$ progressive disease, $P R$ partial response, $S D$ stable disease

${ }^{\mathrm{a}}$ One patient dropped out before placebo administration
We observed that almost all patients who had received ramucirumab prior to nivolumab in ATTRACTION-2 were Japanese (55/57 patients). Therefore, an additional analysis exploring the impact of prior ramucirumab treatment on the efficacy of nivolumab treatment was performed in the Japanese subpopulation. In this analysis, nivolumab showed efficacy in both groups of patients, regardless of prior ramucirumab treatment, compared with placebo. The risks of death and disease progression were numerically better in patients with versus those without prior ramucirumab treatment. This could be attributed to VEGF signaling that is known to modify the tumor immunological environment with T-cell activation and Treg suppression [14-18]. Some reports have also shown positive clinical activity with immune-checkpoint inhibitors and antiangiogenic drugs in lung and renal cancer [23, 24]. This is supported 

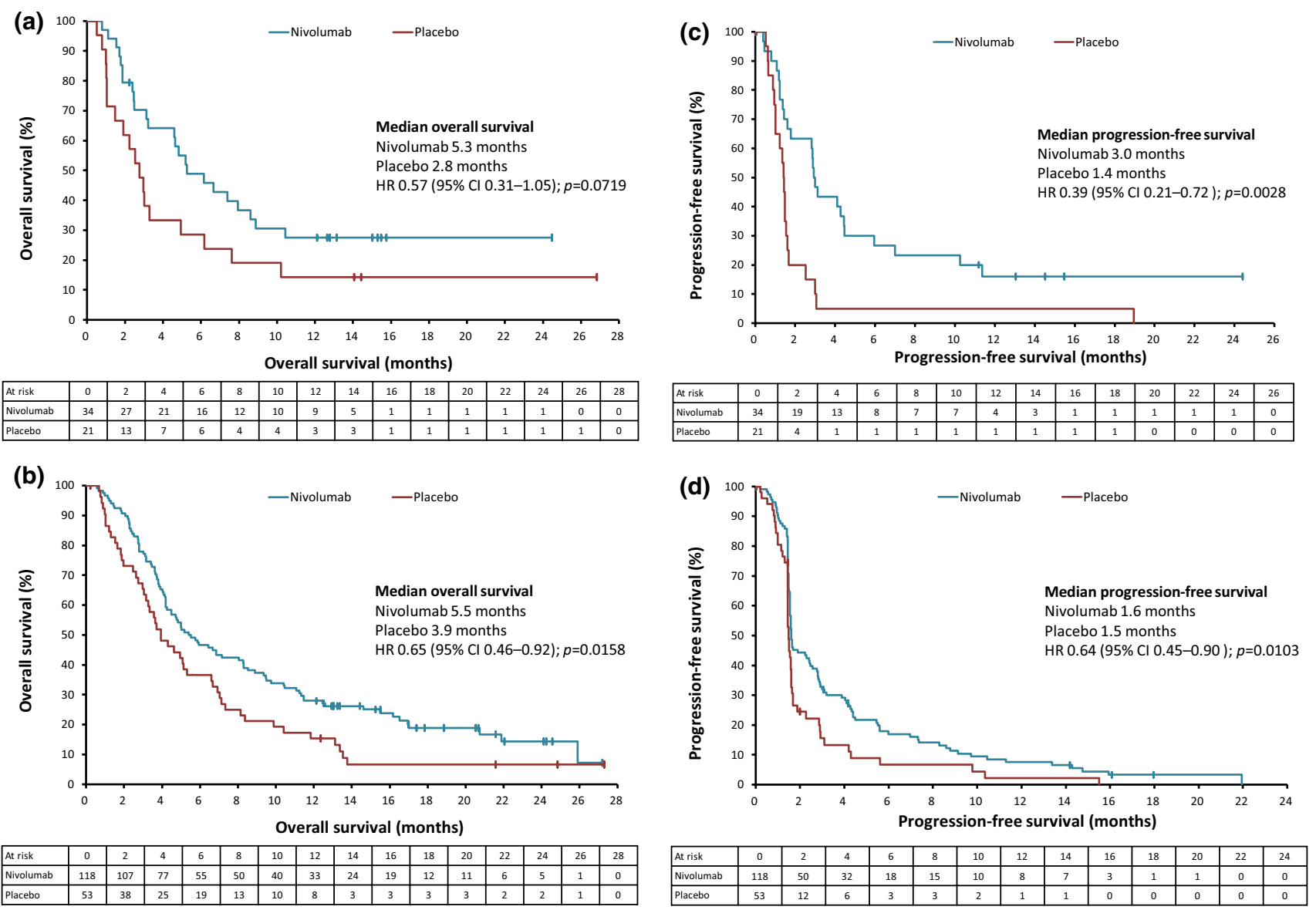

Fig. 2 Kaplan-Meier plots of overall survival in a patients with prior ramucirumab treatment; b patients without prior ramucirumab treatment; and progression-free survival in $\mathbf{c}$ patients with prior ramu-

by the observation that 25 of the 34 patients who received nivolumab directly after ramucirumab treatment reported better clinical efficacy. Therefore, it is speculated that ramucirumab might enhance the efficacy of nivolumab. However, this additional analysis by prior ramucirumab treatment was conducted in a small number of patients. Several clinical trials of the combination with ramucirumab and an anti-PD-1/ PD-L1 antibody are underway, and will help validate our observations.

The median DOR with nivolumab in the Japanese population was 14.5 months (95\% CI 8.3-NA). On the other hand, the DOR with nivolumab in the overall population was reported as 9.5 months (95\% CI 6.14-9.82) [12]. Although the cutoff date was different, the DOR with nivolumab in the Japanese population appears to be longer than that of the overall population. The underlying reason for this is not clear; however, it may be partly attributed to the better performance status in the nivolumab-treated Japanese subpopulation ( $42 \%$ with a performance status of 0 ) compared with the nivolumab-treated overall population

cirumab treatment; d patients without prior ramucirumab treatment (ITT population). CI Confidence interval, $H R$ hazard ratio, ITT intention-to-treat

(29\% with a performance status of 0) reported previously [12].

The safety profile of the Japanese subpopulation was similar to that of the overall study population [12], and no treatment-related AEs specific to the Japanese were observed.

The proportion of PD-L1-positive patients observed in our study was lower than that in the KEYNOTE-059 trial (PD-L1 positivity of 57\%), which employed a combined positive score (including tumor cells, macrophages, and lymphocytes) [25]. This difference in proportion between KEYNOTE-059 and the current study could be due to the difference in the scoring method employed [26]. Moreover, the relationship between the PD-L1 expression score and response to therapy remains to be elucidated. We would also like to acknowledge that treatment efficacy could not be evaluated based on PD-L1 expression due to the limited number of patients in this subgroup analysis. Other biomarkers, including microsatellite instability or Epstein-Barr virus positivity, were not available in this analysis, which may be a limitation of this report. 
Overall, there were no notable differences in the efficacy and safety outcomes with nivolumab between the Japanese and the overall populations, and no treatment-related AEs specific to the Japanese were observed.

\section{Conclusion}

In the Japanese subpopulation, patients with advanced G/ GEJ cancer treated with nivolumab had a manageable safety profile and longer OS, with early and durable responses, versus patients treated with placebo. Additionally, the benefit of sequential use of ramucirumab followed by nivolumab was observed in an exploratory analysis requiring further validation.

Acknowledgements We thank the patients, their families, and the investigators. Editorial support, in the form of medical writing, assembling tables and creating high-resolution images based on authors' detailed directions, collating author comments, copyediting, fact checking, and referencing, was provided by Annirudha Chillar, $\mathrm{MD}, \mathrm{PhD}$, of Cactus Communications, and funded by Ono Pharmaceutical Co., Ltd., Osaka, Japan, and Bristol-Myers Squibb, Princeton, NJ, USA.

Funding This study was funded by Ono Pharmaceutical Co., Ltd., Osaka, Japan, and Bristol-Myers Squibb, Princeton, NJ, USA.

\section{Compliance with ethical standards}

Conflict of interest Ken Kato received research funds from Ono Pharmaceutical, MSD, Shionogi, and Merck Serono for this study, and research funds from Merck, Shionogi, and Ono Pharmaceutical for activities outside the submitted work. Taroh Satoh received research grants and honoraria from Ono Pharmaceutical and Bristol-Myers Squibb and a department donated by Ono Pharmaceutical for this study; research grants and honoraria from Yakult Honsha, Chugai Pharmaceutical, Eli Lilly, Merck Serono, Takeda Pharmaceutical, Taiho Pharmaceutical, and MSD; and a department donated by Yakult Honsha and Chugai Pharmaceutical for activities outside the submitted work. Kei Muro received a grant from Ono Pharmaceutical for this study; grants from Ono Pharmaceutical, MSD, Daiichi Sankyo, Kyowa Hakko Kirin, Shionogi, and Gilead Sciences for activities outside the submitted work; and personal fees from Chugai Pharmaceutical, Taiho Pharmaceutical, Takeda Pharmaceutical, Merck Serono, Eli Lilly, and Yakult Honsha. Takaki Yoshikawa received grants from Ono Pharmaceutical and Bristol-Myers Squibb for this study; grants and honoraria from Chugai Pharmaceutical and Taiho Pharmaceutical; grants and advisory fees from Novartis, Ono Pharmaceutical, Eli Lilly Japan, Johnson \& Johnson, and Covidien; and honoraria from Abbott Japan, Kaken, Yakult Honsha, Nippon Kayaku, Takeda Pharmaceutical, and Olympus for activities outside the submitted work. Takao Tamura received grants from Ono Pharmaceutical and Bristol-Myers Squibb for this study, and grants from MSD Pharmaceutical, Merck, and Daiichi Sankyo for activities outside the submitted work. Keiko Minashi received research funding from Ono Pharmaceutical for this study. Kensei Yamaguchi received grants from Ono Pharmaceutical and Bristol-Myers Squibb for this study. Nozomu Machida received a grant from Ono Pharmaceutical for this study, and grants from Taiho Pharmaceutical, MSD, and Eli Lilly for activities outside the submitted work. Taito Esaki received a grant from Ono Pharmaceutical for this study; grants from Dainippon Sumitomo, Novartis, MSD, Daiichi
Sankyo, Pfizer, Boehringer Ingelheim, Ono Pharmaceutical, Bayer, Astellas, Showa, AstraZeneca, and GSK; personal fees from Eisai, Kyowa Hakko Kirin, Chugai Pharmaceutical, and Bristol-Myers Squibb; and grants and personal fees from Taiho Pharmaceutical, Merck Serono, Eli Lilly, Nippon Kayaku, and Takeda Pharmaceutical for activities outside the submitted work. Masahiro Goto received a grant, personal fees, and non-financial support from Ono Pharmaceutical for this study; grants, personal fees, and non-financial support from Taiho Pharmaceutical, Yakult Honsha, Chugai Pharmaceutical, Kyowa Hakko Kirin, and Mochida Pharmaceutical; and personal fees and nonfinancial support from Takeda Pharmaceutical, Novartis, and Bayer Yakuhin for activities outside the submitted work. Yoshito Komatsu received a grant from Ono Pharmaceutical for this study; lecturer's fee and research expenses from Ono Pharmaceutical, MSD, Eli Lilly, Merck, AstraZeneca, Daiichi Sankyo, Taiho Pharmaceutical, Chugai Pharmaceutical, NCC, Kyowa Hakko Kirin, Takeda Pharmaceutical, Sanofi, Yakult Honsha, Bristol-Myers Squibb, Boehringer Ingelheim, Bayer, Pfizer, and Novartis; and research expenses from Linical and TCOG for activities outside the submitted work. Takako Eguchi Nakajima received a grant from Ono Pharmaceutical for this study; grants and personal fees from Eli Lilly Japan, Taiho Pharmaceutical, Chugai Pharmaceutical, Takeda Pharmaceutical, Merck Serono, Ono Pharmaceutical, and Dainippon Sumitomo; grants from Yakult Honsha, Eisai, Sanofi, Amgen Astellas BioPharma, and AstraZeneca; and personal fees from Sawai Pharmaceutical, Kyowa Hakko Kirin, Bristol-Myers Squibb, Bayer Yakuhin, and Maruho for activities outside the submitted work. Naotoshi Sugimoto received a grant from Ono Pharmaceutical for this study, and grants from Taiho Pharmaceutical, Chugai Pharmaceutical, Eli Lilly, MSD, Daiichi Sankyo, and Dainippon Sumitomo for activities outside the submitted work. Kazuhiro Yoshida received grants, personal fees, and non-financial support from Ono Pharmaceutical for this study; grants, personal fees, and non-financial support from Taiho Pharmaceutical, Chugai Pharmaceutical, Yakult Honsha, Eli Lilly Japan, Daiichi Sankyo, Merck Serono, Novartis Pharma, EA Pharma, Takeda Pharmaceutical, and Sanofi; grants and personal fees from Johnson \& Johnson and Covidien Japan; grants from Nippon Kayaku, Otsuka Pharmaceutical, and Dainippon Sumitomo; and personal fees from MSD K.K. and Bayer Yakuhin for activities outside the submitted work. Eiji Oki received a grant from Ono Pharmaceutical for this study, and honoraria for lecturing from Ono Pharmaceutical, Taiho Pharmaceutical, Yakult Honsha, Merck Serono, Takeda Pharmaceutical, Chugai Pharmaceutical, Eli Lilly, and Bristol-Myers Squibb. Akihito Tsuji received a grant from Ono Pharmaceutical for this study, and honoraria from Daiichi Sankyo, Taiho Pharmaceutical, Chugai Pharmaceutical, Merck Serono, Takeda Pharmaceutical, and Bristol-Myers Squibb Japan for activities outside the submitted work. Mizutomo Azuma received a grant and personal fees from Ono Pharmaceutical for this study. Keisei Taku, Masahiro Tsuda, Tomohiro Nishina, Hirofumi Fujii, Kenji Kunieda, Soh Saito, Yasushi Omuro, Yasuo Iwamoto, Sachio Fushida, Yasuo Hamamoto, and Keisho Chin received grants from Ono Pharmaceutical for this study. Li-Tzong Chen received a grant from Ono Pharmaceutical for this study; grants, personal fees, and non-financial support from Novartis, TTY, and Syncore; grants from the Ministry of Science and Technology (Taiwan), Ministry of Health and Welfare (Taiwan), Pfizer, GSK, Merck Serono, OBI, and Polaris; grants and non-financial support from Celgene; and personal fees from Eli Lilly, PharmaEngine, Shire, MSD, Bristol-Myers Squibb, Ono Pharmaceutical, Five Prime, and Merrimack for activities outside the submitted work. Yoon-Koo Kang received a grant from Ono Pharmaceutical for this study, and personal fees from Ono Pharmaceutical, Bristol-Myers Squibb, Daehwa, and Blueprint for activities outside the submitted work. Narikazu Boku received a grant and personal fees from Ono Pharmaceutical for this study, and personal fees from Chugai Pharmaceutical, Merck Serono, and AstraZeneca for activities outside the submitted work. 
Ethical standards All procedures followed were in accordance with the ethical standards of the responsible committee on human experimentation (institutional and national) and with the Helsinki Declaration of 1964 and later versions.

Informed consent Informed consent to be included in the study, or the equivalent, was obtained from all patients.

Open Access This article is distributed under the terms of the Creative Commons Attribution 4.0 International License (http://creativeco mmons.org/licenses/by/4.0/), which permits unrestricted use, distribution, and reproduction in any medium, provided you give appropriate credit to the original author(s) and the source, provide a link to the Creative Commons license, and indicate if changes were made.

\section{References}

1. Cancer Information Service, National Cancer Center, Japan. Cancer registry and statistics (article in Japanese). 2018. https:// ganjoho.jp/reg_stat/statistics/stat/summary.html. Accessed 16 Apr 2018.

2. Hironaka S, Ueda S, Yasui H, Nishina T, Tsuda M, Tsumura T, et al. Randomized, open-label, phase III study comparing irinotecan with paclitaxel in patients with advanced gastric cancer without severe peritoneal metastasis after failure of prior combination chemotherapy using fluoropyrimidine plus platinum: WJOG 4007 trial. J Clin Oncol. 2013;31:4438-44.

3. Japan Society of Gastric Cancer. Japanese gastric cancer treatment guidelines 2018 (article in Japanese). 2018. http://www.kaneharashuppan.co.jp/books/detail.html?isbn=9784307203814. Accessed 16 Apr 2018.

4. Yamada Y, Higuchi K, Nishikawa K, Gotoh M, Fuse N, Sugimoto N, et al. Phase III study comparing oxaliplatin plus S-1 with cisplatin plus S-1 in chemotherapy-naïve patients with advanced gastric cancer. Ann Oncol. 2015;26:141-8.

5. Koizumi W, Narahara H, Hara T, Takagane A, Akiya T, Takagi M, et al. S-1 plus cisplatin versus S-1 alone for first-line treatment of advanced gastric cancer (SPIRITS trial): a phase III trial. Lancet Oncol. 2008;9:215-21.

6. Sharma P, Allison JP. Immune checkpoint targeting in cancer therapy: toward combination strategies with curative potential. Cell. 2015;161:205-14.

7. Yuan J, Zhang J, Zhu Y, Li N, Tian T, Li Y, et al. Programmed death-ligand-1 expression in advanced gastric cancer detected with RNA in situ hybridization and its clinical significance. Oncotarget. 2016;7:39671-9.

8. Kawazoe A, Kuwata T, Kuboki Y, Shitara K, Nagatsuma AK, Aizawa M, et al. Clinicopathological features of programmed death ligand 1 expression with tumor-infiltrating lymphocyte, mismatch repair, and Epstein-Barr virus status in a large cohort of gastric cancer patients. Gastric Cancer. 2017;20:407-15.

9. Zhang M, Dong Y, Liu H, Wang Y, Zhao S, Xuan Q, et al. The clinicopathological and prognostic significance of PD-L1 expression in gastric cancer: a meta-analysis of 10 studies with 1,901 patients. Sci Rep. 2016;6:37933.

10. Janjigian YY, Bendell JC, Calvo E, Kim JW, Ascierto PA, Sharma $\mathrm{P}$, et al. CheckMate-032: Phase I/II, open-label study of safety and activity of nivolumab (nivo) alone or with ipilimumab (ipi) in advanced and metastatic (A/M) gastric cancer (GC). J Clin Oncol. 2016;34(Suppl 15):4010.

11. Janjigian YY, Ott PA, Calvo E, Kim JW, Ascierto PA, Sharma P, et al. Nivolumab \pm ipilimumab in pts with advanced $(\mathrm{adv}) / \mathrm{meta}-$ static chemotherapy-refractory $(\mathrm{CTx}-\mathrm{R})$ gastric $(\mathrm{G})$, esophageal
(E), or gastroesophageal junction (GEJ) cancer: CheckMate 032 study. J Clin Oncol. 2017;35(Suppl 15):4014.

12. Kang YK, Boku N, Satoh T, Ryu MH, Chao Y, Kato K, et al. Nivolumab in patients with advanced gastric or gastro-oesophageal junction cancer refractory to, or intolerant of, at least two previous chemotherapy regimens (ONO-4538-12, ATTRAC TION-2): a randomised, double-blind, placebo-controlled, phase 3 trial. Lancet. 2017;390:2461-71.

13. National Comprehensive Cancer Network. NCCN Guidelines for Gastric Cancer V.5.2017. 2017. https://www.ncen.org/Common/ FileManager.ashx?fileManagerId=96a310fb-6b85-4233-88170be95e19fc6f. Accessed 16 Apr 2018.

14. Motz GT, Coukos G. The parallel lives of angiogenesis and immunosuppression: cancer and other tales. Nat Rev Immunol. 2011;11:702-11.

15. Della Porta M, Danova M, Rigolin GM, Brugnatelli S, Rovati B, Tronconi C, et al. Dendritic cells and vascular endothelial growth factor in colorectal cancer: correlations with clinicobiological findings. Oncology. 2005;68:276-84.

16. Takahashi A, Kono K, Ichihara F, Sugai H, Fujii H, Matsumoto Y. Vascular endothelial growth factor inhibits maturation of dendritic cells induced by lipopolysaccharide, but not by proinflammatory cytokines. Cancer Immunol Immunother. 2004;53:543-50.

17. Liu XD, Hoang A, Zhou L, Kalra S, Yetil A, Sun M, et al. Resistance to antiangiogenic therapy is associated with an immunosuppressive tumor microenvironment in metastatic renal cell carcinoma. Cancer Immunol Res. 2015;3:1017-29.

18. Wallin JJ, Bendell JC, Funke R, Sznol M, Korski K, Jones S, et al. Atezolizumab in combination with bevacizumab enhances antigen-specific T-cell migration in metastatic renal cell carcinoma. Nat Commun. 2016;7:12624.

19. Eisenhauer EA, Therasse P, Bogaerts J, Schwartz LH, Sargent $\mathrm{D}$, Ford R, et al. New response evaluation criteria in solid tumours: revised RECIST guideline (version 1.1). Eur J Cancer. 2009;45:228-47.

20. National Cancer Institute. Common Terminology Criteria for Adverse Events (CTCAE) version 4.0. 2010. https://evs.nci.nih. gov/ftp1/CTCAE/CTCAE_4.03/Archive/CTCAE_4.0_2009-0529_QuickReference_8.5x11.pdf. Accessed 16 Apr 2018.

21. Shitara K, Muro K, Shimada Y, Hironaka S, Sugimoto N, Komatsu Y, et al. Subgroup analyses of the safety and efficacy of ramucirumab in Japanese and Western patients in RAINBOW: a randomized clinical trial in second-line treatment of gastric cancer. Gastr Cancer. 2016;19:927-38.

22. Ohtsu A, Shah MA, Van Cutsem E, Rha SY, Sawaki A, Park $\mathrm{SR}$, et al. Bevacizumab in combination with chemotherapy as first-line therapy in advanced gastric cancer: a randomized, double-blind, placebo-controlled phase III study. J Clin Oncol. 2011;29:3968-76.

23. Socinski MA, Jotte RM, Cappuzzo F, Orlandi F, Stroyakovskiy D, Nogami N, et al. Atezolizumab for first-line treatment of metastatic nonsquamous NSCLC. N Engl J Med. 2018;378:2288-301.

24. McDermott DF, Huseni MA, Atkins MB, Motzer RJ, Rini BI, Escudier B, et al. Clinical activity and molecular correlates of response to atezolizumab alone or in combination with bevacizumab versus sunitinib in renal cell carcinoma. Nat Med. 2018;24:749-57.

25. Fuchs CS, Doi T, Jang RW, Muro K, Satoh T, Machado M, et al. Safety and efficacy of pembrolizumab monotherapy in patients with previously treated advanced gastric and gastroesophageal junction cancer: phase 2 clinical KEYNOTE-059 trial. JAMA Oncol. 2018;4:e180013.

26. Kulangara K, Zhang N, Corigliano E, Guerrero L, Waldroup S, Jaiswal D, et al. Clinical utility of the combined positive score for programmed death ligand-1 expression and the approval of pembrolizumab for treatment of gastric cancer. Arch Pathol Lab 
Med. 2018. https://doi.org/10.5858/arpa.2018-0043-OA. (Epub

ahead of print).

\section{Affiliations}

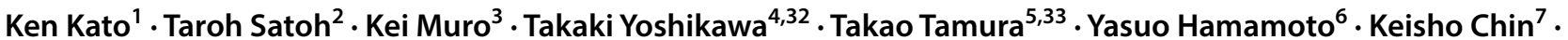
Keiko Minashi $^{8} \cdot$ Masahiro Tsuda $^{9} \cdot K^{2}$ ensei Yamaguchi ${ }^{10,34}$. Nozomu Machida ${ }^{11}$ - Taito Esaki ${ }^{12}$. Masahiro Goto ${ }^{13}$. Yoshito Komatsu ${ }^{14} \cdot$ Takako Eguchi Nakajima $^{15} \cdot$ Naotoshi Sugimoto $^{16} \cdot$ Kazuhiro Yoshida $^{17} \cdot$ Eiji Oki $^{18}$. Tomohiro Nishina ${ }^{19} \cdot$ Akihito Tsuji $^{20,35} \cdot$ Hirofumi Fujii $^{21} \cdot K^{2}$ ji Kunieda ${ }^{22} \cdot$ Soh Saitoh $^{23} \cdot$ Yasushi Omuro $^{24}$. Mizutomo Azuma ${ }^{25}$. Yasuo Iwamoto ${ }^{26} \cdot$ Keisei Taku $^{27}$ - Sachio Fushida ${ }^{28} \cdot$ Li-Tzong Chen $^{29,30}$ - Yoon-Koo Kang ${ }^{31}$. Narikazu Boku ${ }^{1}$

1 Division of Gastrointestinal Medical Oncology, National Cancer Center Hospital, 5-1-1, Tsukiji, Chuo-ku,

Tokyo 104-0045, Japan

2 Department of Frontier Science for Cancer and Chemotherapy, Osaka University Graduate School of Medicine, Suita, Japan

3 Department of Clinical Oncology, Aichi Cancer Center Hospital, Nagoya, Japan

4 Department of Gastrointestinal Surgery, Kanagawa Cancer Center, Yokohama, Japan

5 Department of Medical Oncology, Kindai University, Faculty of Medicine, Osaka, Japan

6 Keio Cancer Center, School of Medicine, Keio University, Tokyo, Japan

7 Department of Gastroenterology, Cancer Institute Hospital of the Japanese Foundation for Cancer Research, Tokyo, Japan

8 Clinical Trial Promotion Department, Chiba Cancer Center, Chiba, Japan

9 Department of Gastroenterological Oncology, Hyogo Cancer Center, Akashi, Japan

10 Department of Gastroenterology, Saitama Cancer Center, Saitama, Japan

11 Division of Gastrointestinal Oncology, Shizuoka Cancer Center, Shizuoka, Japan

12 Department of Gastrointestinal and Medical Oncology, National Hospital Organization Kyushu Cancer Center, Fukuoka, Japan

13 Cancer Chemotherapy Center, Osaka Medical College Hospital, Takatsuki, Japan

14 Division of Cancer Chemotherapy, Hokkaido University Hospital Cancer Center, Sapporo, Japan

15 Department of Clinical Oncology, St. Marianna University School of Medicine, Kawasaki, Japan

16 Department of Medical Oncology, Osaka International Cancer Institute, Osaka, Japan

17 Department of Surgical Oncology, Gifu University Graduate School of Medicine, Gifu, Japan

18 Department of Surgery and Science, Graduate School of Medical Sciences, Kyushu University, Fukuoka, Japan
19 Department of Gastrointestinal Medical Oncology, National Hospital Organization Shikoku Cancer Center, Matsuyama, Japan

20 Department of Medical Oncology, Kobe City Medical Center General Hospital, Kobe, Japan

21 Department of Clinical Oncology, Cancer Center Jichi Medical University Hospital, Shimotsuke, Japan

22 Department of Medical Oncology, Saku Central Hospital Advanced Care Center, Saku, Japan

23 Internal Medicine, Misawa City Hospital, Misawa, Japan

24 Department of Medical Oncology, Tokyo Metropolitan Cancer and Infectious Diseases Center Komagome Hospital, Tokyo, Japan

25 Department of Gastroenterology, Kitasato University School of Medicine, Sagamihara, Japan

26 Medical Oncology, Hiroshima City Hiroshima Citizens Hospital, Hiroshima, Japan

27 Medical Oncology, Shizuoka General Hospital, Shizuoka, Japan

28 Gastroenterological Surgery, Kanazawa University Hospital, Kanazawa, Japan

29 National Institute of Cancer Research, National Health Research Institutes, Tainan, Taiwan, Republic of China

30 Department of Internal Medicine, National Cheng Kung University Hospital, National Cheng Kung University, Tainan, Taiwan, Republic of China

31 Department of Oncology, Asan Medical Center, University of Ulsan College of Medicine, Seoul, South Korea

32 Present Address: Department of Gastric Surgery, National Cancer Center Hospital, Tokyo, Japan

33 Present Address: Department of Medical Oncology, Kindai University Nara Hospital, Ikoma, Japan

34 Present Address: Department of Gastroenterology, Cancer Institute Hospital of the Japanese Foundation for Cancer Research, Tokyo, Japan

35 Present Address: Department of Medical Oncology, Kagawa University, Takamatsu, Japan 